VoL. 54 (1996) [1-3]

\title{
ADMISSIBLE LIMITS OF BLOCH FUNCTIONS ON BOUNDED STRONGLY PSEUDOCONVEX DOMAINS
}

\author{
Hu Zhanguian
}

\begin{abstract}
Let $\mathcal{D} \subseteq \mathbb{C}^{n}$ be a bounded strongly pseudoconvex domain with $C^{2}$ boundary $\partial \mathcal{D}$. In this paper we prove that for a Bloch function in $\mathcal{D}$ the existance of radial limits at almost all $\zeta \in \partial D$ imphes the existence of admissible limits almost everywhere on $\partial \mathcal{D}$.
\end{abstract}

Let $\mathcal{D} \subseteq \mathbb{C}^{n}$ be a bounded strongly pseudoconvex domain with $C^{2}$ boundary $\partial \mathcal{D}$ (for the definition we refer to [1]). For $z \in \mathcal{D}$ the Euclidean distance from $z$ to $\partial \mathcal{D}$ is denoted by $\delta(z)$ and for $\zeta \in \partial \mathcal{D}$ the unit outward normal of $\partial \mathcal{D}$ at $\zeta$ is denoted by $v_{\zeta}$. If $\zeta \in \partial \mathcal{D}$ and $\alpha>0$ we define the admissible approach region $\mathcal{U}_{\alpha}(\zeta)$ with the vertex $\zeta$ by

$$
\mathcal{U}_{\alpha}(\zeta)=\left\{z \in \mathcal{D} ;\left|(z-\zeta) \cdot \bar{v}_{\zeta}\right|<(1+\alpha) \delta_{\zeta}(z),|z-\zeta|^{2}<\alpha \delta_{\zeta}(z)\right\},
$$

where $\delta_{\zeta}(z)=\min \left\{\delta(z), \operatorname{dist}\left(z, T_{\zeta}(\partial \mathcal{D})\right)\right\}$ and $T_{\zeta}(\partial \mathcal{D})$ is the real tangent space to $\partial \mathcal{D}$ at $\zeta$. A function $f$ on $\mathcal{D}$ is called to have an admissible limit at $\zeta$ if $\lim _{z \rightarrow \zeta, z \in \mathcal{U}_{\alpha}(\zeta)} f(z)$ exists for all $\alpha>0$.

Let $\mathcal{B}(\mathcal{D})$ be the space of all Bloch functions $f$ which are holomorphic in $\mathcal{D}$ with $\sup \{|\nabla f(z)| \cdot \delta(z): z \in \mathcal{D}\}<\infty$. It is well known that each function in $H^{p}$ has an admissible limit at almost every $\zeta \in \partial D$. But for Bloch functions generally we can say nothing on the existence of admissible limits. For example, Ullrich constructed a Bloch function in the unit ball (a typical model of a strongly pseudoconvex domain) in $\mathbb{C}^{n}$ which has a radial limit at no point of the boundary (see [2]). On the other hand, Lehto and Virtanen proved in [3] that the existence of radial limits implies the existence of angular limits for Bloch functions in the unit disc $D=\{z \in \mathbb{C}:|z|<1\}$.

The purpose of this paper is to extend this result to the setting of strongly pseudoconvex domains. Our approach will be very different from that of [3] and our result also generalises [4].

Received 21 August 1995

Research supported by the Natural Science Foundation of Zhejiang Province.

Copyright Clearance Centre, Inc. Serial-fee code: 0004-9729/96 \$A2.00+0.00. 
THEOREM. Let $\mathcal{D} \subseteq \mathbb{C}^{n}$ be a bounded strongly pseudoconvex domain with $C^{2}$ boundary, and let $f$ be a Bloch function in $\mathcal{D}$. If the limit $\lim _{t \rightarrow+0} f\left(\zeta-t v_{\zeta}\right)$ exists at almost all $\zeta \in \partial \mathcal{D}$, then $f$ has admissible limits almost everywhere on $\partial \mathcal{D}$.

Proof: First, we do some estimates on $\mathcal{U}_{\alpha}(\zeta)$ for $\zeta \in \partial \mathcal{D}$. Without loss of the generality we may assume that $\zeta$ is the origin, that $v_{\zeta}$ is in the negative $y_{1}$ direction (here $z_{1}=x_{1}+y_{1} i$ ) and thus the complex normal space $\mathcal{N}_{\zeta}$ and the complex tangential space $\mathcal{T}_{\zeta}$ are

$$
\mathcal{N}_{\zeta}=\left\{\left(z_{1}, 0, \ldots, 0\right) ; z_{1} \in \mathbb{C}\right\}, \mathcal{T}_{\zeta}=\left\{\left(0, z_{2}, \ldots, z_{n}\right) ; z_{j} \in \mathbb{C}, j=2, \ldots, n\right\} .
$$

For $z=\left(z_{1}, \ldots, z_{n}\right) \in \mathcal{U}_{\alpha}(\zeta)$, by definition

$$
\begin{aligned}
& \left|z_{1}\right|=\left|\left\langle z-\zeta, v_{\zeta}\right\rangle\right|<(1+\alpha) y_{1}, \\
& \left|z_{2}\right|^{2}+\cdots+\left|z_{n}\right|^{2} \leqslant|z-\zeta|^{2}<\alpha y_{1} .
\end{aligned}
$$

Put $z^{\prime}=\left(y_{1} i, 0, \ldots, 0\right)$ and let $P_{z^{\prime}}\left(r_{1}, r_{2}\right)$ be the polydisc centred at $z^{\prime}$ with radius $r_{1}$ in the complex normal direction and $r_{2}$ in each complex tangential direction [1, p.55]. Then we know from (1) that $z \in \mathcal{U}_{\alpha}(\zeta)$ implies $z \in P_{z^{\prime}}\left(\sqrt{3 \alpha} y_{1}, \sqrt{\alpha} \sqrt{y_{1}}\right)$. Notice that Lemma 6 of [5] is still valid under the weaker assumption that $\mathcal{D}$ is a bounded strongly pseudoconvex domain with $C^{2}$ boundary in $\mathbb{C}^{n}$, with the same proof to the case of $C^{\infty}$ boundary. By applying this lemma we can take $\alpha>0$ so small that

$$
P_{z^{\prime}}\left(\sqrt{3 \alpha} y_{1}, \sqrt{\alpha} \sqrt{y_{1}}\right) \subseteq\left\{w \in \mathcal{D} ; \beta\left(z^{\prime}, w\right)<1\right\}
$$

where $\beta\left(z^{\prime}, w\right)$ is the Kobayashi distance from $z^{\prime}$ to $w$. Hence, for $z \in \mathcal{U}_{\alpha}(\zeta) \cap\{w$ : $|w-\zeta|<\varepsilon\}$ with $\varepsilon$ small enough we have

$$
\beta\left(z^{\prime}, z\right)<1 .
$$

Now suppose $f \in \mathcal{B}(\mathcal{D})$ and for almost all $\zeta \in \partial \mathcal{D}$ the limit $\lim _{t \rightarrow+0} f\left(\zeta-t v_{\zeta}\right)$ exists. Set $E=\left\{\zeta \in \partial \mathcal{D}: \lim _{t \rightarrow+0} f\left(\zeta-t v_{\zeta}\right)\right.$ exists $\}$. If $\zeta \in E$, then $f$ is bounded on $\left\{\zeta-t v_{\zeta}: 0<t \leqslant \varepsilon\right\}$, say

$$
\left|f\left(\zeta-t v_{\zeta}\right)\right| \leqslant M \quad t \in(0, \varepsilon] .
$$

Then for $z \in \mathcal{U}_{\alpha}(\zeta) \cap\{w:|w-\zeta|<\varepsilon\}$, from the estimate on [6, p.150] and (2), (3) we obtain

$$
\begin{aligned}
|f(z)| & \leqslant\left|f(z)-f\left(z^{\prime}\right)\right|+\left|f\left(z^{\prime}\right)\right| \\
& \leqslant C \beta\left(z^{\prime}, z\right)+M \leqslant C+M .
\end{aligned}
$$

That $f$ is bounded on $\mathcal{U}_{\alpha}(\zeta) \backslash\{w:|w-\zeta|<\varepsilon\}$ is obvious. This means that $f$ is admissible bounded at $\zeta \in E$. Theorem 12 of [1] tells us that $f$ has admissible limits at almost all $\zeta \in \partial \mathcal{D}$. The proof is complete. 
REMARK. We have actually proved the following: If $E \subseteq \partial \mathcal{D}$ is measurable and $f$ is a Bloch function which has radial limits at each $\zeta \in E$, then $f$ has admissible limits at almost all $\zeta \in E$.

\section{REFERENCES}

[1] E.M. Stein, Boundary behavior of holomorphic functions of several complex variables (Princeton University Press, Princeton, NJ, 1972).

[2] D.C. Ullrich, 'A Bloch function in the ball with no radial limits', Bull. London Math. Soc. 20 (1988), 337-341.

[3] O. Lehto and K.J. Virtanen, 'Boundary behavior and normal meromorphic functions', Acta Math. 97 (1959), 47-65.

[4] Hou Xiangdong, 'Bloch functions on the unit ball', Chinese Ann. Math. Ser. A 8 (1987), 287-299.

[5] H. $\mathrm{Li}$, 'BMO, VMO and Hankel operators on the Bergman space of strongly pseudoconvex domains', J. Funct. Anal. 106 (1992), 375-408.

[6] S.G. Krantz and D. Ma, 'Bloch functions on strongly pseudoconvex domains', Indiana Univ. Math. J. 37 (1988), 145-163.

\footnotetext{
Department of Mathematics

Huzhou Teachers College

Huzhou, Zhejiang 313000

People's Republic of China
} 